\title{
Fabrication and characterization of single carbon nanotube emitters as point electron sources
}

\author{
Gongpu Zhao, Jian Zhang, Qi Zhang, Han Zhang, Otto Zhou, and Lu-Chang Qin ${ }^{\text {a) }}$ \\ Department of Physics and Astronomy, University of North Carolina at Chapel Hill, Chapel Hill, \\ North Carolina 27599-3255 and Curriculum in Applied and Materials Sciences, \\ University of North Carolina at Chapel Hill, Chapel Hill, North Carolina 27599-3255 \\ Jie Tang \\ National Institute for Materials Science, Tsukuba 305-0047, Japan
}

(Received 22 February 2006; accepted 4 October 2006; published online 8 November 2006)

\begin{abstract}
Individual carbon nanotube electron field emitters with good controllability have been fabricated in a two-step process involving (a) producing micron-size carbon fibers which contain single carbon nanotubes at their cores by a chemical vapor deposition method and (b) exposing the nanotubes by fracturing the fiber with mechanical forces and mounting the fiber to a copper wire. These fiber-nanotube electron emitters show low operating voltage, generate high field enhancement, and produce fine electron beams. The field emission characteristics and durability of this structure offer promising applications for analytical instruments where high performance point electron sources are required. (C) 2006 American Institute of Physics. [DOI: 10.1063/1.2387961]
\end{abstract}

Carbon nanotubes (CNTs) exhibit excellent properties in electrical field-induced electron emission with high brightness, stable emission current, long lifetime, and narrow energy distribution. ${ }^{1-3}$ Furthermore, the strong $s p^{2} \mathrm{C}-\mathrm{C}$ bonds result in that carbon nanotubes can withstand strong electric fields during the field emission process. Carbon nanotubes also have a high aspect ratio and small radius, which will assist the nanotubes to emit electrons at a relatively low electric field. These excellent properties make CNTs a promising candidate to outperform the contemporary tungsten cold field emission point electron source.

Though many efforts have been devoted to make largearea carbon-nanotube-based cathodes, there still lacks a simple and precise method to mount one single CNT to a metal probe tip to obtain a point electron emitter. ${ }^{4-6}$ In this work, we present a simple, reproducible, and efficient method to fabricate single nanotube electron emitters with good controllability. The fabrication is a two-step process involving (a) producing micron-size carbon fibers that contain single nanotubes at their cores by a chemical vapor deposition method $^{7}$ and (b) exposing the nanotubes and attaching the fiber to a support structure. At the cross section of the fractured carbon fiber, a multiwalled nanotube sticks out due to its different fracture toughness from the carbon fiber that is largely made of amorphous carbon. The protruded carbon nanotubes are usually $4-30 \mathrm{~nm}$ in diameter and a few tens of nanometers to several microns in length.

Figure 1(a) shows schematically the process by which a carbon fiber was fractured, picked up, and aligned with the metal support. After the fiber was attached to the copper ribbon and well aligned, the entire structure was examined in a transmission electron microscope (TEM, JEOL 2010F) using a custom-designed sample holder. Figure 1(b) shows the morphology of an individual multiwalled carbon nanotube (CNT 3) extruded from the carbon fiber. The length and diameter of each CNT have been measured and are listed in Table I.

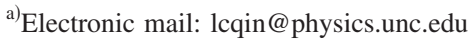

The field emission pattern of CNT 3 showed a ringlike structure suggesting that this nanotube had an open end. ${ }^{8,9}$ Ideally, electrons emitted from an open-ended nanotube are radially accelerated and form a circular ring on the phosphor screen. To further support this, we have measured the emission patterns from both CNT 2 with an open end [Fig. 2(a)] and a capped CNT [Fig. 2(b)]. The corresponding emission patterns are shown in Figs. 2(c) and 2(d), respectively. It clearly shows that the electrons emitted from an open CNT form a ring pattern and, on the other hand, electrons emitted from a capped CNT form a solid spot. The TEM images also revealed that the nanotube is well crystallized, attributed to the high synthesis temperature. It is usually desirable to have a well-crystallized nanotube in order to obtain an emitter of long emission lifetime. ${ }^{10}$

To obtain a carbon nanotube of desired length to improve the spatial coherence of the point emitter, ${ }^{11}$ we used the electron beam of $0.7 \mathrm{~nm}$ in diameter to cut the nanotube to a length of less than $1 \mu \mathrm{m}$. Figure 2(e) shows the TEM

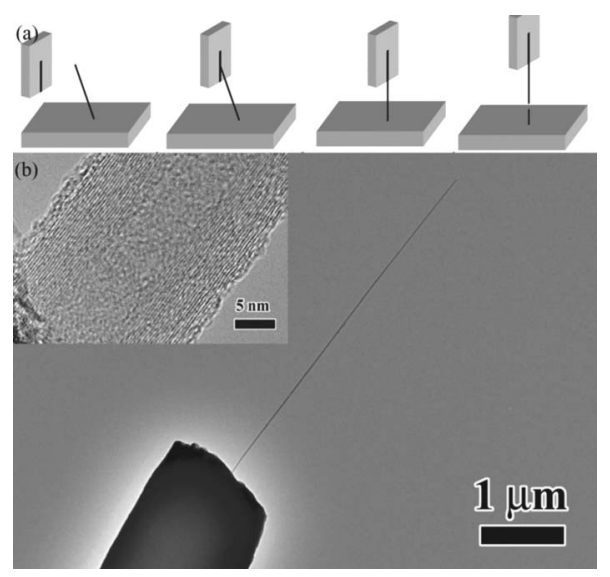

FIG. 1. (a) Schematic depicting the process by which a carbon fiber is fractured, picked up, and aligned with a copper ribbon, which has a precarved groove on it. (b) Morphology of CNT 3 of Table II, in which a multiwalled carbon nanotube (TEM image shown in inset) extrudes from a fractured carbon fiber. 
TABLE I. Length $L$, diameter $D$, and angle $\theta$ relative to the axis of the support structure for ten mounted individual carbon nanotubes.

\begin{tabular}{cccc}
\hline \hline Mounted MWNT & $L(\mu \mathrm{m})$ & $D(\mathrm{~nm})$ & $\theta(\mathrm{deg})$ \\
\hline 1 & 0.7 & 7.3 & 2 \\
2 & 0.5 & 12 & 4 \\
3 & 4.4 & 24 & 5 \\
4 & 1.1 & 8 & 9 \\
5 & 1.4 & 6 & 1 \\
6 & 1.1 & 4 & 4 \\
7 & 0.5 & 5.5 & 14 \\
8 & 0.3 & 6.5 & 8 \\
9 & 1.4 & 10 & 13 \\
10 & 2.6 & 11 & 3 \\
\hline \hline
\end{tabular}

image of a CNT that was cut by the electron beam.

Field emission measurements were carried out on CNTs 1-3 in a vacuum chamber operated at $10^{-7}$ torr. CNT 2, shown in Fig. 2(a), has an open end obtained by electron beam cutting. The emission currents of these CNTs were measured as functions of the extraction voltage [Fig. 3(a)] and the corresponding Fowler-Nordheim plots are given in Fig. 3(b). In order to further remove the adsorbed species, ${ }^{9,12,13}$ we heated CNT 4 at $1200{ }^{\circ} \mathrm{C}$ for 3 min using a tungsten heating filament in a vacuum of $3 \times 10^{-8}$ torr. $^{9}$ The Fowler-Nordheim plots both before and after cleaning are shown in Fig. 3(c).

The Fowler-Nordheim theory correlates the emission current density $J$ and the electric field $F$ for an emitter of work function $\phi{ }^{14}$

$$
\begin{aligned}
J(T & =0, F, \phi) \\
& =1.5 \times 10^{-6} \frac{F^{2}}{\phi} \exp \left[\frac{10.4}{\phi^{1 / 2}}-\frac{6.44 \times 10^{7} \phi^{3 / 2}}{F}\right],
\end{aligned}
$$

and the field enhancement factor $\beta$ is defined by

$$
F=\beta V,
$$

where $V$ is the applied extraction voltage. The experimental data exhibited a linear relationship on the Fowler-Nordheim

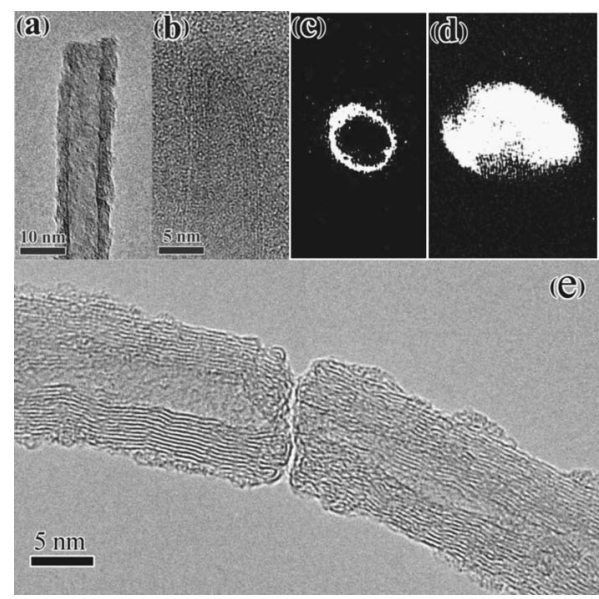

FIG. 2. (a) TEM image of CNT 2 listed in Table II which has an open end obtained by electron beam cutting. (b) TEM image of a capped CNT. (c) Electron emission pattern from the CNT indicating that the electrons emitted from an open CNT form a ring pattern. (d) Electron emission pattern from a capped CNT shown in Fig. 3(b). (e) TEM image showing a multiwalled carbon nanotube cut by a $200 \mathrm{kV}$ electron beam of $0.7 \mathrm{~nm}$ in diameter. (a)

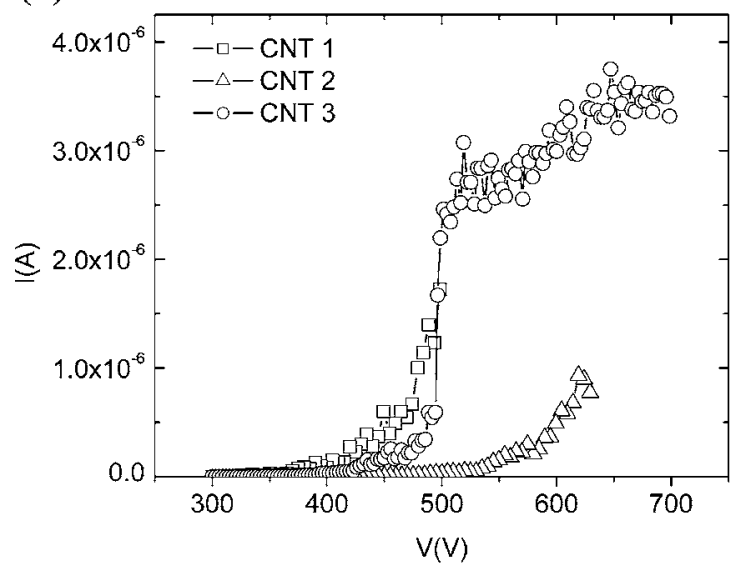

(b)

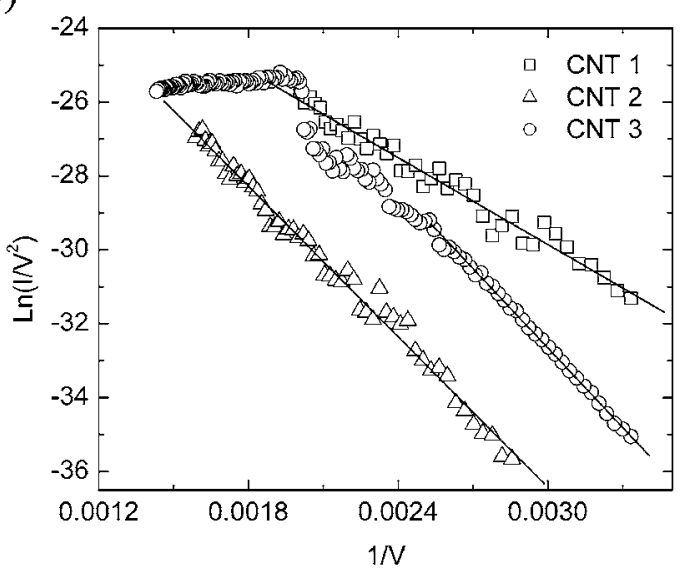

(c)

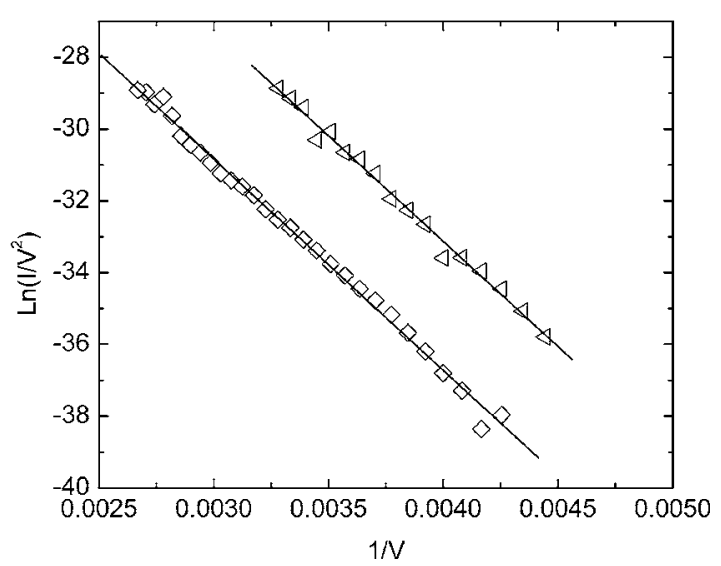

FIG. 3. (a) Field emission measurement of three individual multiwalled nanotubes (MWNTs) (CNTs 1-3) at room temperature. The emission current was measured as a function of the extraction voltage. CNT 3 is shown in Fig. 1(b). (b) The corresponding Fowler-Nordheim plots and linear fits to the Fowler-Nordheim plots. (c) Fowler-Nordheim plots for CNT 4 before (triangle) and after (square) cleaning.

plot $\left[\ln \left(I / V^{2}\right)\right.$ vs $\left.V^{-1}\right]$ at low current up to $30 \mathrm{nA}$. A fit to the Fowler-Nordheim equation using the work function $\phi$ $=5.1 \mathrm{eV},{ }^{15-17}$ the value of the field enhancement factor $\beta$, and the effective emitting area $A$ have been calculated and are listed in Table II. Besides the errors introduced from curve fitting, another source of errors is due to the uncertainties of the work function which is $\pm 0.2 \mathrm{eV} .^{16}$ The uncertainties have also been calculated and are listed in Table II. From the $I-V$ curve, the minimum electric field needed to extract $10 \mathrm{nA}$ current is $1.1 \mathrm{~V} / \mu \mathrm{m}$. Saturation of the emission cur- 
TABLE II. Field emission data from four individual carbon nanotubes (CNTS 1-4) of length $L$, diameter $D$, field enhancement factor $\beta$, and emission area $A$.

\begin{tabular}{ccccc}
\hline \hline $\begin{array}{c}\text { CNT } \\
\text { No. }\end{array}$ & $L(\mu \mathrm{m})$ & $D(\mathrm{~nm})$ & $\begin{array}{c}\beta / 10^{7}\left(\mathrm{~m}^{-1}\right) \\
\phi=5.1 \pm 0.2 \mathrm{eV}\end{array}$ & $\begin{array}{c}A\left(\mathrm{~m}^{2}\right) \\
\phi=5.1 \pm 0.2 \mathrm{eV}\end{array}$ \\
\hline 1 & 0.5 & 24 & $1.9 \pm 0.2$ & $(1.3 \pm 0.4) \times 10^{-18}$ \\
2 & 0.5 & 12 & $1.09 \pm 0.09$ & $(3.2 \pm 0.7) \times 10^{-17}$ \\
3 & 4.4 & 24 & $1.04 \pm 0.07$ & $(4.3 \pm 0.1) \times 10^{-15}$ \\
4 & 1.1 & 8 & $1.2 \pm 0.1$ & $(3 \pm 2) \times 10^{-14}$ \\
4 (cleaned) & 1.1 & 8 & $1.2 \pm 0.1$ & $(1.4 \pm 0.9) \times 10^{-14}$ \\
\hline \hline
\end{tabular}

rent from the single CNTs at $3-4 \mu \mathrm{A}$ was usually observed in our experiment.

For an isolated emitter with a hemispherical tip on a cylinder, $\beta=1 / 5 r$ is a good approximation for the field enhancement factor where $r$ is the radius of curvature of the tip. ${ }^{18}$ Although this approximation has been used to estimate the radius of curvature, it was found to be invalid for the carbon nanotube emitters with a carbon fiber support. If we use this approximation, the radius of CNT 3 is estimated to be $20 \mathrm{~nm}$ and this is nearly two times of the actual radius $(12 \mathrm{~nm})$ of the carbon nanotube measured in the TEM images. This discrepancy is attributed to two reasons: (a) the nanotube measured here has an open end and (b) the field enhancement from the micron-size carbon fiber was not considered.

For the model structure that has a nanotube standing between two planar electrodes with separation $d$, the electric field at the nanotube tip can be expressed as $F=\gamma_{\mathrm{CNT}} F_{0}$, with $F_{0}=V / d$ and $\gamma_{\mathrm{CNT}}$ being a factor that depends on the geometrical attributes of the nanotube such as the cap structure, length $L$, and radius $r .^{19,20}$ For a capped carbon nanotube ${ }^{19}$ $\gamma_{\mathrm{CNT}}=1.2(2.15+L / r)^{0.9}$, and for an open carbon nanotube ${ }^{20}$ $\gamma_{\mathrm{CNT}}=0.62 L / r+0.14 L / w+7$, where $w$ is the wall thickness.

To account for the effects on the field enhancement of the carbon fiber, here we have used a cylinder with a flat top and of the same diameter as the carbon fiber as the cathode on which a carbon nanotube is standing. The electric field at the tip of the carbon nanotube standing between a cylindrical cathode and a planar anode can be described by a multistage model, ${ }^{21}$

$$
F=\gamma_{\mathrm{CNT}} \gamma_{\text {fiber }} \frac{V}{d}=\gamma_{\mathrm{CNT}} F_{M},
$$

and the field enhancement factor $\beta$ can be expressed as

$$
\beta=F / V=\gamma_{\mathrm{CNT}} F_{M} / V,
$$

where $F_{M}$ is the reference electric field along the cylinder axis when the nanotube is absent. Since the length of the carbon nanotube is comparable to the diameter of carbon fiber, the electric field along the nanotube can vary more than one order of magnitude. We therefore chose $F_{M}$ as the local electric field at the tip of the carbon nanotube. On the other hand, $F_{M}$ also depends strongly on the diameter of the carbon fiber, the nanotube length $L$, and interelectrode distance $d$. We deduced the $\beta$ factor by calculating $F_{M}$ using the experimental parameters and observed excellent agreement with the experimental data listed in Table II. ${ }^{22}$
Although Eq. (3) is an empirical formula, it is also physically appealing. Firstly, the $F_{M} / V$ term is independent of $V$, as confirmed by the calculations, and $\beta$ factor is not sensitive to the applied voltage between the electrodes. Secondly, $F_{M} / V$ takes into account both the geometry of the fiber and the interelectrode distance, which make the $\beta$ factor vary with the interelectrode distance $d$. This agrees well with the experimental results. ${ }^{21,23}$ Thirdly, the calculations also show that when $L$ increases, $F_{M}$ will decrease. Although in experiment the nanotube can have a wide range of lengths, since $F_{M}$ increases inversely as $h$, this makes the field enhancement factor of the fiber-CNT emitter vary within a relatively small range, which makes this structure more reproducible in field enhancement.

In summary, we have established a method to fabricate single nanotube field emitters. This method is simple and easy to control. These single nanotube emitters exhibit excellent field emission properties such as low operating voltage and high field enhancement. An empirical formula has also been developed to calculate the field enhancement factor for an open-end nanotube attached on a carbon fiber support.

The authors wish to thank financial support from Xintek/ DOE SBIR and one of the authors (J.T.) is partially supported by the JSPS Japan-US Collaborative Scientific Program.

${ }^{1}$ N. de Jonge, Y. Lamy, K. Schoots, and T. H. Oosterkamp, Nature (London) 420, 393 (2002).

${ }^{2}$ M. J. Fransen, Th. L. Van Rooy, and P. Kruit, Appl. Surf. Sci. 146, 312 (1999).

${ }^{3}$ J. M. Bonard, J. P. Salvetat, T. Stöckli, L. Forró, and A. Châtelain, Appl. Phys. A: Mater. Sci. Process. 69, 245 (1999).

${ }^{4}$ N. de Jonge, Y. Lamy, and M. Kaiser, Nano Lett. 3, 1621 (2003).

${ }^{5}$ J. H. Hafner, C. L. Cheung, T. H. Oosterkamp, and C. M. Lieber, J. Phys. Chem. B 105, 743 (2001).

${ }^{6}$ J. Tang, G. Yang, Q. Zhang, A. Parhat, B. Maynor, J. Liu, L.-C. Qin, and O. Zhou, Nano Lett. 5, 11 (2005).

${ }^{7}$ L.-C. Qin and S. Iijima, Mater. Lett. 30, 311 (1997).

${ }^{8}$ Y. Saito, K. Hamaguchi, K. Hata, K. Uchida, Y. Tasaka, F. Ikazaki, M. Yumura, A. Kasuya, and Y. Nishina, Nature (London) 389, 554 (1997).

${ }^{9}$ K. Hata, A. Takakura, and Y. Saito, Surf. Sci. 490, 296 (2001).

${ }^{10}$ Y. Wei, C. Xie, K. A. Dean, and B. F. Coll, Appl. Phys. Lett. 79, 4527 (2001).

${ }^{11}$ H. Schmid and H.-W. Fink, Appl. Phys. Lett. 70, 2679 (1997).

${ }^{12}$ K. A. Dean and B. R. Chalamala, J. Appl. Phys. 85, 3832 (1999).

${ }^{13}$ K. A. Dean and B. R. Chalamala, Appl. Phys. Lett. 76, 375 (2000).

${ }^{14}$ I. Brodie and C. A. Spindt, Advances in Electronics and Electron Physics (Academic, San Diego, 1992), 83, 11.

${ }^{15}$ R. Gao, Z. Pan, and Z. L. Wang, Appl. Phys. Lett. 78, 1757 (2001).

${ }^{16} \mathrm{~N}$. de Jonge, M. Allioux, M. Doytcheva, M. Kaiser, K. B. K. Teo, R. G. Lacerda, and W. I. Milne, Appl. Phys. Lett. 85, 1607 (2004).

${ }^{17}$ O. Gröning, O. M. Küttel, Ch. Emmenegger, P. Gröning, and L. Schlapbach, J. Vac. Sci. Technol. B 18, 665 (2000).

${ }^{18}$ R. Gomer, Field Emission and Field Ionization (Harvard University Press, Cambridge, MA, 1961), p. 32.

${ }^{19}$ R. G. Forbes, C. J. Edgcombe, and U. Valdrè, Ultramicroscopy 95, 57 (2003).

${ }^{20}$ G. C. Kokkorakis, J. A. Roumeliotis, and J. P. Xanthakis, J. Appl. Phys. 95, 1468 (2004).

${ }^{21}$ J. Y. Huang, K. Kempa, S. H. Jo, S. Chen, and Z. F. Ren, Appl. Phys. Lett. 87, 053110 (2005).

${ }^{22}$ G. Zhao, Ph.D. thesis, University of North Carolina at Chapel Hill, 2006.

${ }^{23}$ J. M. Bonard, K. A. Dean, B. F. Coll, and C. Klinke, Phys. Rev. Lett. 89, 197602 (2002). 